\title{
Clinical experiences in fungal keratitis caused by Acremonium
}

This article was published in the following Dove Press journal:

Clinical Ophthalmology

23 January 2014

Number of times this article has been viewed

\author{
Seong-Jae Kim ${ }^{1,2}$ \\ Yong-Wun Cho' \\ Seong-Wook Seo ${ }^{1,2}$ \\ Sun-Joo Kim ${ }^{2,3}$ \\ Ji-Myong Yoo ${ }^{1,2}$
}

'Department of Ophthalmology, ${ }^{2}$ Gyeongsang Institute of Health Science, ${ }^{3}$ Department of Laboratory Medicine, Gyeongsang National University, College of Medicine, Jinju, Korea
Correspondence: Seong-Jae Kim Department of Ophthalmology, School of Medicine, Institute of Health Science, Gyeongsang National University, Jinju, Gyeongnam 660-75I, S Korea

Tel + 82557508170

Fax +82557584158

Email mayal2kim@naver.com
Purpose: To report the predisposing risk factors, clinical presentation, management, and therapeutic outcomes of fungal keratitis caused by Acremonium.

Methods: This is a retrospective study of cases with Acremonium fungal keratitis that presented to our tertiary referral center between January 2006 and August 2012. Patient demographic and clinical details were determined and reported.

Results: Five cases of fungal keratitis from Acremonium species were identified in five patients (three males, two females). The mean age of the patients was of $73.4 \pm 5.46$ years, with a mean follow-up time of $124 \pm 72$ days. All patients had a history of corneal trauma with vegetable matter. Four cases were unresponsive to initial treatment ( $0.2 \%$ fluconazole, $0.15 \%$ amphotericin B) and required topical 5\% natamycin, and, in two out of five cases, topical $1 \%$ voriconazole.

Conclusion: The most common risk factors for Acremonium fungal keratitis was ocular trauma. When a corneal lesion is found to be unresponsive to the initial treatment, we should consider adding or substituting topical natamycin or voriconazole for treatment.

Keywords: Acremonium, fungal keratitis, natamycin, prognosis, voriconazole

\section{Introduction}

Fungal keratitis is an unusual but potentially sight-threatening ocular infection. The majority of fungal keratitis cases occur after corneal injury, usually as a result of contact with a fungus-contaminated plant material. A broad spectrum of fungal species have been identified as causative agents, and it is important to identify the causative agent of keratitis correctly to treat the patient effectively. ${ }^{1}$

Acremonium are filamentous fungi commonly isolated from plant debris and soil. ${ }^{2}$ They appear as hyaline, septate mycelial elements with branched or intertwined hyphae. Acremonium are an opportunistic environmental pathogen that lead to a superficial infection. ${ }^{3,4}$ Das et $\mathrm{al}^{5}$ reported a total of 17 cases between 1971 and 2010 of worldwide clinical ocular infections caused by different species of Acremonium, which described symptoms including keratitis and endophthalmitis in these patients. Alfonso et $\mathrm{al}^{6}$ reported four cases of Acremonium fungal keratitis associated with laser-assisted in situ keratomileusis (LASIK) that were performed by different surgeons during the same time period in the same operating room. Aside from these instances, until recently, there had been no other reported case series of Acremonium fungal keratitis.

This retrospective study describes patients admitted for treatment of Acremonium fungal keratitis in a tertiary referral center. We believe that this report may provide some useful information on the diagnosis and management of Acremonium fungal keratitis. 


\section{Methods}

We reviewed the medical records of all patients with Acremonium fungal keratitis who were treated at the Gyeongsang National University Hospital (GNUH) between January 2006 and August 2012. Demographic characteristics and clinical results of total cases are summarized Table 1. Patients were included in the study when the culture results obtained by corneal scraping showed a positive result. A total of five cases were enrolled in this study.

On admission, corneal ulcers were scraped with a scalpel blade and the culture specimens were obtained by scraping the base and margins of the ulcer. These specimens were placed onto slides for Gram staining and potassium hydroxide $(\mathrm{KOH})$ staining according to standard protocols. Furthermore, specimen samples were inoculated onto blood, chocolate, MacConkey, and Sabouraud agar plate surfaces for culture. All bacterial and fungal isolates were identified using standard laboratory techniques.

All patients were treated with standard antibiotics immediately after admission and prior to the culture results, which consisted of hourly instillations of topical fourth-generation quinolones (moxifloxacin or gatifloxacin). If a fungal infection was identified through $\mathrm{KOH}$ staining, an antifungal course was initiated, consisting of hourly applications of topical $0.2 \%$ fluconazole and $0.15 \%$ amphotericin B with oral fluconazole. We altered the therapy (topical natamycin or voriconazole) on the basis of the clinical response for each patient. Initial treatment failure was defined by an increase in the size of the ulcer or infiltration, or impending perforation. In cases where this adjusted treatment failed, conjunctival flap surgery was performed.

\section{Results}

\section{Patient demographic and clinical data}

From January 2006 and August 2012, 260 patients with infectious keratitis were admitted to the GNUH. Of these patients, five patients (three males, two females), with a mean age of $73.4 \pm 5.46$ years were identified as having Acremonium fungal keratitis. The mean duration from the onset of symptoms to time of admission was $6.6 \pm 2.30$ days. And the mean follow-up time was $4 \pm 2$ months. Visual acuity (VA) on presentation ranged from 0.02 to 0.2 . At the time of the last follow-up, VA had improved in all patients.

\section{Case I}

A 64-year old woman injured her left eye with vegetable matter. On examination, biomicroscopy revealed a peripheral corneal epithelial defect, which was surrounded by ring-shaped stromal infiltration with a feathery border (Figure 1A). Corneal scraping showed hyphae. The patient was started on an hourly instillation of topical $0.5 \%$ moxifloxacin (Vigamox; Alcon Laboratories, Inc., Fort Worth, TX, USA), 0.15\% amphotericin B (Ambisome, Yuhan Corporation, Seoul, Korea), 0.2\% fluconazole (Diflucan, Ilyang Pharmaceutical, Seoul, Korea), and oral fluconazole (Oneflu, Joongwea Shinyak, Seoul, Korea), $100 \mathrm{mg}$ twice daily. After 1 week, there was no reduction in the size of the corneal infiltrate (Figure 1B). Corneal cultures were subsequently positive for Acremonium (Figure 1C and D). The patient was lost to follow-up, but returned 2 weeks later with worsening of the infiltrate and a large epithelial defect (Figure 1E). The cornea was re-scraped to improve the penetration of the topical treatment. Despite hourly treatment with topical eye drops, the patient's corneal condition failed to improve. We added topical 5\% natamycin (Natacyn; Alcon) to the regime. After 2 days, we observed a marked reduction in the size of the infiltrate. Topical amphotericin B and natamycin were gradually tapered over the next 5 weeks. At the final visit, her best-corrected visual acuity (BCVA) was 0.5 , and there was no recurrence over the following 6 months (Figure 1F).

\section{Case 2}

A 76-year old man complaining of severe ocular pain in his left eye was referred to our hospital. He had a history of trauma by plant material. Slit-lamp biomicroscopy showed a central corneal infiltrate that was associated with a large epithelial defect and hypopyon (Figure 2A). The patient was started on an hourly instillation of topical $0.5 \%$ moxifloxacin, and intravenous ciprofloxacin, twice daily. After 5 days on

Table I Patient demographic and clinical data

\begin{tabular}{|c|c|c|c|c|c|c|}
\hline Case & $\begin{array}{l}\text { Age } \\
\text { (years) }\end{array}$ & Sex & $\begin{array}{l}\text { Duration from onset of } \\
\text { symptoms to diagnosis (days) }\end{array}$ & $\begin{array}{l}\text { Follow-up period } \\
\text { (days) }\end{array}$ & $\begin{array}{l}\text { Initial visual } \\
\text { acuity }\end{array}$ & $\begin{array}{l}\text { Final visual } \\
\text { acuity }\end{array}$ \\
\hline I & 64 & Female & 7 & 180 & 0.2 & 0.5 \\
\hline 2 & 76 & Male & 5 & 180 & 0.1 & 0.5 \\
\hline 3 & 75 & Male & 10 & 120 & 0.2 & 0.5 \\
\hline 4 & 78 & Male & 7 & 60 & 0.02 & 0.15 \\
\hline 5 & 74 & Female & 4 & 60 & 0.02 & 1.0 \\
\hline
\end{tabular}




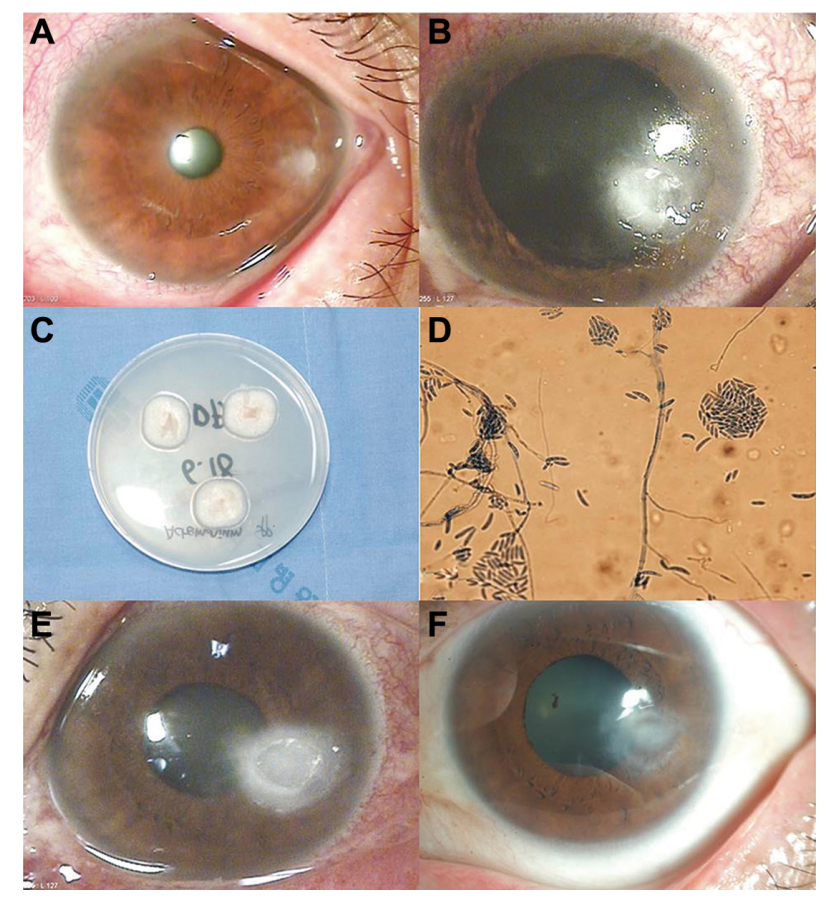

Figure I (A) Case I. At initial examination, a slit-lamp photograph showed a peripheral corneal epithelial defect with a ring-shaped stromal infiltration. (B) Stromal infiltration had increased after I week. (C) Colony formation on Sabouraud's dextrose agar exhibited a white, powdery, suede-like appearance. (D) Lactophenol cotton blue mounting of Acremonium spp. (magnification, 400x). (E) After 4 weeks, the case showed worsening of the infiltrate and a large epithelial defect. (F) After 6 months, biomicroscopy showed complete resolution, with a faint scar.

this therapy, the condition of the left eye worsened with the appearance of an endothelial plaque. Repeated corneal smears showed septate fungal hyphae. The patient was administered with hourly doses of $0.15 \%$ amphotericin B and $0.2 \%$ fluconazole, with oral fluconazole. Over the next 7 days, corneal cultures were positive for Acremonium. Topical 5\% natamycin and 1\% voriconazole (Vfend; Pfizer Pharmaceuticals Korea, Seoul, Korea) with oral fluconazole were prescribed immediately. The final VA was 0.5 , and there was no recurrence in the following 6 months (Figure 2B).

\section{Case 3}

A 75-year old male patient was injured in his left eye with vegetable matter. On slit-lamp examination of the left eye, there was a central white stromal infiltrate with corresponding epithelial defect and endothelial plaque (Figure 2C). Corneal smears showed hyphae. The patient was started on an hourly instillation of topical $0.5 \%$ moxifloxacin, $0.15 \%$ amphotericin $\mathrm{B}$, $0.2 \%$ fluconazole, and oral fluconazole. After 7 days on this therapy, the size of the infiltrate was markedly reduced, and the hypopyon had disappeared. Corneal cultures were subsequently positive for Acremonium. The final BCVA was 0.5 , and there was no recurrence in the following 4 months (Figure 2D).

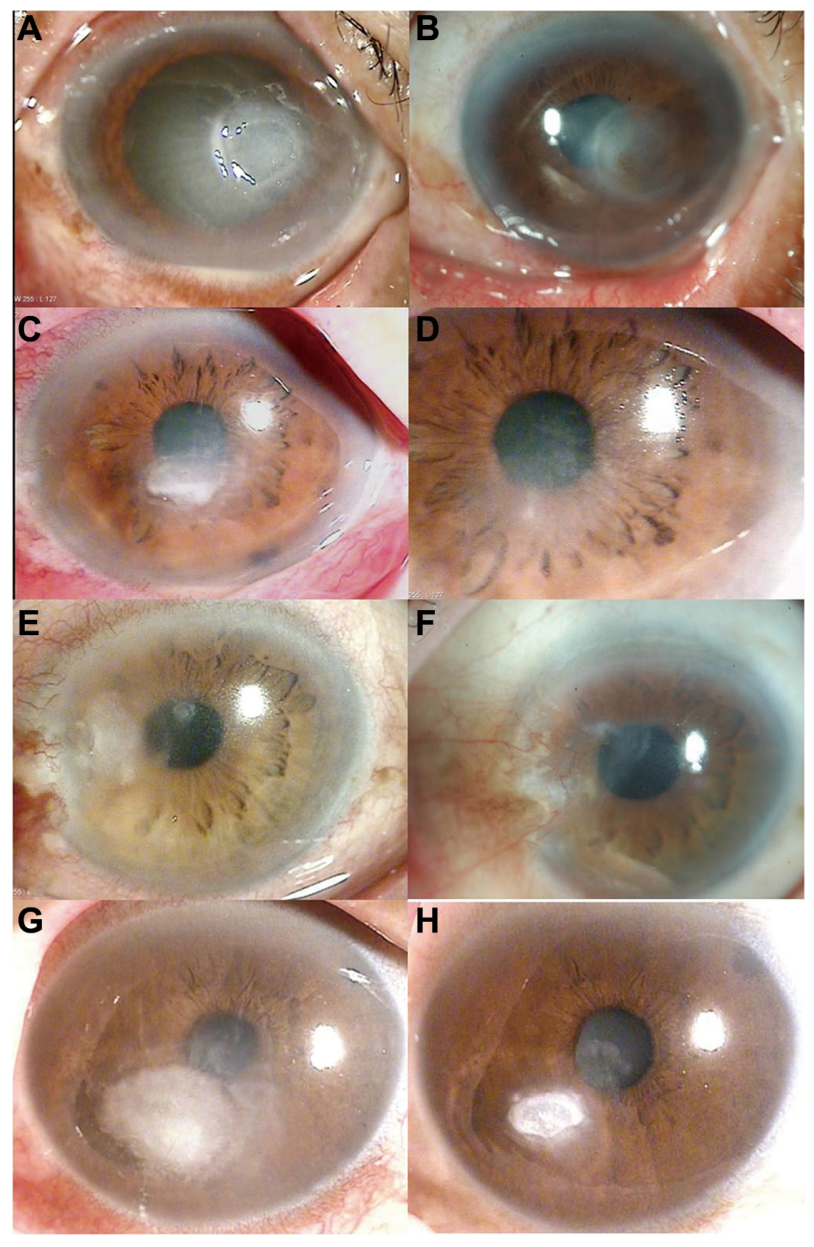

Figure 2 (A) Case 2. At initial examination, a slit-lamp photograph showed a central corneal infiltrate with a large epithelial defect and hypopyon. (B) After 6 months, biomicroscopy showed complete resolution, with a faint scar. (C) Case 3 . At initial examination, a slit-lamp photograph showed a central stromal infiltrate with endothelial plaque and hypopyon. (D) After 4 months, biomicroscopy showed complete resolution, with a faint scar. (E) Case 4. At initial examination, a slit-lamp photograph showed mild conjunctival injection and a peripheral corneal infiltrate. (F) After 3 months, biomicroscopy showed complete resolution, with a faint scar and a peripheral conjunctival flap remnant. (G) Case 5. At initial examination, a slitlamp photograph showed a paracentral stromal infiltrate with an endothelial plaque. (H) After 2 months, biomicroscopy showed resolution of the corneal infiltrate.

\section{Case 4}

A 78-year old man complaining of decreased vision in his left eye was referred to our clinic. He had been injured in this eye with vegetable matter. Slit-lamp biomicroscopy showed mild conjunctival injection and a peripheral corneal infiltrate was associated with an epithelial defect in the left eye (Figure 2E). Corneal smears showed hyphae. He was started on an hourly instillation of topical $0.5 \%$ moxifloxacin, $0.15 \%$ amphotericin B, $0.2 \%$ fluconazole, and oral fluconazole. After 2 days of therapy, the clinical feature of the left eye had worsened, with the appearance of new stromal infiltrates. Topical application of 5\% natamycin was included in his treatment regime. Seven days later, Acremonium was isolated from 
corneal culture. Despite this treatment, the patient's condition failed to improve and the cornea was melting around the limbus. The treatment was changed to topical 5\% natamycin and $1 \%$ voriconazole, and oral voriconazole, $200 \mathrm{mg}$ twice a day. After 13 days of therapy, the patient underwent conjunctival flap surgery due to a progressive thinning of the cornea. After surgery, the corneal lesion was improved and topical natamycin and voriconazole were gradually tapered. The final BCVA was 0.15 , and there was no recurrence in the following 2 months (Figure 2F).

\section{Case 5}

A 74-year old woman was injured in her right eye with grass. On slit-lamp examination of the right eye, there was a paracentral whitish stromal infiltrate with an overlying epithelial defect and an endothelial plaque (Figure 2G). Corneal smears showed hyphae. She was started on an hourly regimen of topical $0.5 \%$ moxifloxacin, $0.15 \%$ amphotericin B, $0.2 \%$ fluconazole, 5\% natamycin and oral fluconazole. Seven days after corneal culture, the organism was identified as Acremonium. The patient's final VA was 1.0, and there was no recurrence in the following 2 months (Figure $2 \mathrm{H}$ ).

\section{Discussion}

Acremonium is a large polyphyletic fungal genus that comprises approximately 150 species, most of them being saprobes in soil and pathogens of plants. ${ }^{7,8}$ The majority of the cases of Acremonium infections presented thus far have been caused by species $A$. recifei, $A$. strictum, $A$. kiliense, $A$. reseogriseum, A. alabamensis, $A$. falciform, and $A$. potronii. ${ }^{9}$ In most cases, Acremonium is diagnosed with microscopic morphology. The typical morphological features of Acremonium include slowgrowing colonies, thin hyphae, and long, narrow, and tapered phialides formed singly as shown in Figure 1D. In addition, the phialides produces conidia which are small, mostly unicellular, in slimy heads, chains, or both. ${ }^{10,11}$ Species of Acremonium are morphologically very similar to each other and at best can only be distinguished on the basis of subtle differences, making their identification difficult. Therefore, in most of the clinical cases the etiological agent is reported only as an Acremonium species. To confirm Acremonium species, deoxyribonucleic acid (DNA)-based method is essential but technical problems like no defined reference sequence and cost and time limitations makes this method difficult. ${ }^{12}$ Therefore, this report also has the limitation that Acremonium species was not confirmed via the DNA-based method.

Acremonium can be easily isolated on modified Sabouraud's dextrose agar at a temperature of $25^{\circ} \mathrm{C}-37^{\circ} \mathrm{C}$ (optimum $30^{\circ} \mathrm{C}$ ). Colony characteristics of Acremonium vary according to their growth, ranging from white, powdery, and suede-like colonies to smooth, waxy, and velvety colonies, with color variations from white to gray to rose, displaying light yellow or light pink on the reverse side of the culture plate after 4-5 days of incubation. Microscopically, Acremonium form delicate, thin hyaline septate hyphae with inter-twining ropes, and bear narrow, unbranched tapering conidiophores at right angles to the hyphae, which have elliptical or crescentshaped unicellular conidia. ${ }^{2,11}$ These mycological characteristics are remarkably consistent with the findings in our five patients.

Filamentous fungi, like Acremonium, are common environmental saprophytes that cause a variety of infections mostly thought to be secondary to prior colonization and increased host susceptibility. Thus corneal trauma from an insult with vegetable matter is the leading risk factor for the development of fungal keratitis in tropical regions or developing countries. In the present case series, all five immunocompetent patients presented with a history of corneal trauma with contaminated vegetable matter. Besides, all of our patients were an advanced age and lived in rural areas. Therefore, these demographic factors may also influence host susceptibility to corneal trauma and subsequent infection.

Management of fungal corneal ulcers can be difficult, particularly as there are no standard therapies for infections caused by Acremonium. Nowadays, topical and oral voriconazole have been reported to be effective for the treatment of fungal keratitis, but some cases do not respond to treatment with voriconazole, so new antifungal agents, like caspofungin, represent alternatives. ${ }^{13}$ Furthermore, these drugs were too expensive to prescribe for the initial treatment of fungal keratitis. Especially in Korea, fungal keratitis has limitations of treatment in terms of using natamycin and voriconazole due to its cost and insurance problem. Thus, this case series used amphotericin B and fluconazole as initial treatments. From several studies, ${ }^{12,14}$ Acremonium is known to be sensitive to amphotericin B, natamycin, and voriconazole, but initial treatment with amphotericin B and fluconazole failed in four cases out of five patients in our study. Therefore, natamycin or voriconazole might be a better choice of treatments for Acremonium fungal keratitis than amphotericin B or fluconazole.

Despite the limitations of a small sample size and the fact that the DNA-based method was not conducted, and no antibiotics sensitivity test was performed, we believe that 
our results are clinically significant and may provide some useful information on the diagnosis and management of Acremonium fungal keratitis.

In conclusion, based on our findings, the most common risk factors for Acremonium fungal keratitis is ocular trauma, and Acremonium fungal keratitis might be treated better with natamycin and voriconazole than alternatives.

\section{Disclosure}

The authors report no conflicts of interest in this work.

\section{References}

1. Ou JI, Acharya NR. Epidemiology and treatment of fungal corneal ulcers. Int Ophthalmol Clin. 2007;47(3):7-16.

2. Fincher RM, Fisher JF, Lovell RD, Newman CL, Espinel-Ingroff A, Shadomy HJ. Infection due to the fungus Acremonium (cephalosporium). Medicine (Baltimore). 1991;70(6):398-409.

3. Anadolu R, Hilmioğlu S, Oskay T, Boyvat A, Peksari Y, Gürgey E. Indolent Acremonium strictum infection in an immunocompetent patient. Int J Dermatol. 2001;40(7):451-453.

4. Lopes JO, Kolling LC, Neumaier W. Kerionlike lesion of the scalp due to Acremonium kiliense in a noncompromised boy. Rev Inst Med Trop Sao Paulo. 1995;37(4):365-368.
5. Das S, Saha R, Dar SA, Ramachandran VG. Acremonium species: a review of the etiological agents of emerging hyalohyphomycosis. Mycopathologia. 2010;170(6):361-375.

6. Alfonso JF, Baamonde B, Santos J, et al. Acremonium fungal infection in 4 patients after laser in situ keratomileusis. J Cataract Refract Surg. 2004;30:262-272.

7. Zaias N. Superficial white onychomycosis. Sabouraudia. 1966;5: 99-103.

8. Schinabeck MK, Ghannoum MA. Human hyalohyphomycoses: a review of human infections due to Acremonium spp., Paecilomyces spp., Penicillium spp., and Scopulariopsis spp. J Chemother. 2003; 15 Suppl 2:5-15.

9. Walsh TJ, Groll AA, Hiemenz J, et al. Infections due to emerging and uncommon medically important fungal pathogens. Clin Microbiol Infect. 2004;10:48-66.

10. Domsch KH, Fisher JF, Lovell RD, et al. Acremonium in Compendium of Soil Fungi. Germany: IHW-Verlag, Eching; 2007:30-38.

11. Summerbell RC. Aspergillus, Fusarium, Sporothrix, Piedraia. And their relatives. In: Howard DH, editor. Pathogenic Fungi in Humans and Animals. 2nd ed. New York: Marcel Dekker; 2003:237-498.

12. Perdomo H, Sutton DA, García D, et al. Spectrum of clinically relevant Acremonium species in the United States. J Clin Microbiol. 2011;49(1): $243-256$.

13. Hurtado-Sarrió M, Duch-Samper A, Cisneros-Lanuza A, Díaz-Llopis M, Peman-Garcíia J, Vazquez-Polo A. Successful topical application of caspofungin in the treatment of fungal keratitis refractory to voriconazole. Arch Ophthalmol. 2010;128(7):941-942.
Clinical Ophthalmology

\section{Publish your work in this journal}

Clinical Ophthalmology is an international, peer-reviewed journal covering all subspecialties within ophthalmology. Key topics include: Optometry; Visual science; Pharmacology and drug therapy in eye diseases; Basic Sciences; Primary and Secondary eye care; Patien Safety and Quality of Care Improvements. This journal is indexed on

Submit your manuscript here: http://www.dovepress.com/clinical-ophthalmology-journal

\section{Dovepress}

PubMed Central and CAS, and is the official journal of The Society of Clinical Ophthalmology (SCO). The manuscript management system is completely online and includes a very quick and fair peer-review system, which is all easy to use. Visit http://www.dovepress.com/ testimonials.php to read real quotes from published authors. 